\title{
Health impact of dietary neoformed Maillard products in young healthy adults: occurrence in heat-treated foods and bioavailability
}

\author{
G. Saavedra ${ }^{1}$, L. Ait-Ameur ${ }^{1}$, F. Tessier ${ }^{1}$, P. Pouillart ${ }^{1}$, J. M. Lecerf ${ }^{2}$ and I. Birlouez-Aragon ${ }^{1}$ \\ ${ }^{1}$ Institut Polytechnique LaSalle Beauvais, Beauvais, France and ${ }^{2}$ Institut Pasteur, Lille, France
}

Dietary Maillard products (MP) may exert both a positive and a negative health impact ${ }^{(1)}$. Neither the bioavailability nor the global health effect in healthy subjects of MP produced in common heat-treated foods in the Western diet are known, which impedes the evaluation of the related risk $^{(2)}$.

The aim of the present study was to: quantify the exposure to MP, including carboxymethyllysine (CML), fluorescent MP (FMP) and acrylamide, in a normal diet with conventional cooking methods, including grilling, roasting and frying and in a diet of steam-cooked food; assess the contribution of different foods to total exposure; evaluate CML and FMP bioavailability.

Sixty-two non-smoking healthy volunteers were assigned randomly to 4 weeks on a normal diet (high MP) and a steam-cooked nutritionally-equivalent (low MP) diet in a cross-over design. MP content was evaluated chemically in the complete diet and in some specific foods, as well as in faeces, plasma and urine. CML was quantified using GC-MS/MS ${ }^{(3)}$ and FMP were determined from the parallel factor analysis decomposition of 3-D spectra derived from diluted samples (biological samples and mixed meal supernatant fractions) or directly from the mixed meal utilizing a front-face acquisition mode ${ }^{(4)}$.

With the normal diet $500 \mu \mathrm{g}$ acrylamide and $6 \mathrm{mg}$ CML were absorbed, but replacing conventional cooking by steam cooking or minimal processing resulted in a 2-fold-5-fold decrease in absorption. The same fluorophore composition was observed in the meals, the faeces and the urine, but not in fasting plasma. A comparison of the biological levels after the two diets indicated a 10-15\% absorption rate and approximately $100 \%$ CML and FMP urinary excretion rates. De novo synthesis of CML and FMP from absorbed dietary substrates was suggested by the $10 \%$ decrease $(P<0.001)$ in plasma protein-bound CML and FMP after the steam-cooked diet compared with the normal diet. The metabolic impact of endogenous exposure to MP is presented elsewhere ${ }^{(5)}$.

The present study is the first to quantify precisely the dietary exposure to various MP derived from a normal diet, as well as the lowering effect of steam cooking. The biovailability of the most common MP is also clearly demonstrated for food prepared conventionally. Correlations between biological MP levels and metabolic variables suggest a rapid and significant negative bioactivity of such compounds. It can be concluded that steam cooking is an efficient way of reducing exposure and improving many metabolic variables, including insulin resistance index, TAG and long-chain $n-3$ fatty acids. The specific MP involved and the mechanism remain to be elucidated.

1. Somoza V (2005) Mol Nutr Food Res 49, 663-672.

2. Pouillart P, Mauprivez H, Ait-Ameur L, Cayzeele A, Lecerf JM, Tessier F \& Birlouez-Aragon I (2008) Ann NY Acad Sci (In the Press).

3. Charissou A, Ait-Ameur L \& Birlouez-Aragon I (2007) J Chromatogr A 1140, 189-194.

4. Rizkallah J, Lakhal L \& Birlouez-Aragon I (2008) In Optical Methods for Monitoring Fresh and Processed Food-Basics and Applications for a Better Understanding of Non-Destructive Sensing (In the Press).

5. Lecerf JM, Cayzeele A, Vaysse C, Galinier A, Pouillart P, Tessier F \& Birlouez-Aragon I (2007) Proc Nutr Soc 67, OCE, E191 